\title{
The Rolling Earlobe Flap for Dilated Ear Holes Following Ear Gauging: A Novel Approach to Aesthetically Preserving Earlobe Soft Tissue Volume
}

\author{
Wan-Sze Pek ${ }^{1}$, Lin Hon Terence Goh ${ }^{1}$, Chong Han Pek ${ }^{2}$ \\ ${ }^{1}$ Department of Plastic, Reconstructive and Aesthetic Surgery, Singapore General Hospital, Singapore; ${ }^{2}$ Plastic, Reconstructive and Aesthetic \\ Surgery Section, Department of General Surgery, Tan Tock Seng Hospital, Singapore
}

Patients are increasingly seeking repair of their earlobes following ear gauging. Research has shown that current repair techniques either excessively reduce the lobular volume or leave an obvious scar along the free edge of the earlobe. In our case series, we describe the use of a novel technique for repairing earlobes following ear gauging using a rolling earlobe flap that preserves the lobular volume and avoids leaving a scar on the free edge of the lobule. The procedure was performed on 3 patients ( 6 earlobes) who had defects from ear gauging that ranged from 3.0 to $6.5 \mathrm{~cm}$. There were no postoperative complications of infection, wound dehiscence, flap necrosis, hypertrophic scars, or keloids, and all patients were highly satisfied with the postoperative results. This versatile technique allows for an aesthetically pleasing reconstruction of the lobule with the advantages of: the absence of a surgical scar on the free edge of the lobule, preserving the lobule volume, and presenting a highly customizable technique that allows lobules to be created with various shapes and volumes.

Keywords Ear deformities, acquired / Body piercing / Surgical flaps
Correspondence: Chong Han Pek Plastic, Reconstructive and Aesthetic Surgery Section, Department of General Surgery, Tan Tock Seng Hospital, 11 Jalan Tan Tock Seng, Singapore 308433 , Singapore Tel: $+65-91396903$

E-mail: pekch@rocketmail.com

This article was presented as the Plastic \& Aesthetic Surgery Meeting 2016 on Sep 2-3 in Singapore.

No potential conflict of interest relevant to this article was reported.

Received: 19 Dec $2016 \bullet$ Revised: 11 Jun 2017 • Accepted: 27 Jun 2017

pISSN: 2234-6163 • elSSN: 2234-6171 • https://doi.org/10.5999/aps.2017.44.5.453• Arch Plast Surg 2017;44:453-456

\section{INTRODUCTION}

"Plug and tunnel" ear gauges have risen in popularity as a fash-
ion trend, in which sequentially larger ear "plugs" are used to in-
crease the diameter of the dilated ear holes. Despite the popular-
ity of these ear gauges during adolescence, those with piercings
often seek earlobe repair later in life due to professional, familial,
or societal pressures. In Asia, full and plump earlobes are a sym-
bol of wealth and prosperity. Therefore, restoring dilated ear
holes back to socially aesthetic norms presents a surgical chal-
lenge. The current techniques found in the literature include methods to repair dilated ear holes using a variety local flaps or tissue rearrangements [1-8]. These techniques, despite being acceptable forms of repair, often fall short in aesthetic outcomes, as they either leave undesirable scars on the free edge of the lobule or significantly reduce the earlobe volume. Contraction of a scar that extends over the free edge of the lobule often leads to notching of the earlobe and ultimately an undesirable lobule shape.

Based on a review of the current literature on the various methods of dilated earlobe repair, we aim to describe a simple, yet versatile flap without the shortcomings of existing techniques. 
We also designed a local flap that was based on the vascular supply of the external ear and used to achieve closure of dilated ear holes in 3 patients, which resulted in a desirable aesthetic outcome without any complications.

\section{IDEA}

\section{Anatomical basis of the flap design}

The earlobe is supplied by perforators that arise from the posterior aspect of the posterior auricular artery and the superficial temporal artery, anteriorly. In a non-dilated earlobe, innumerable perforators form a dense network of blood vessels that supplies the lobule of the auricle $[9,10]$. Cadaveric dissections have also revealed that this dense network of blood vessels is closely aligned with the free edge of the lobule. This supports our theory that the lobule in a dilated ear hole, although stretched and thinned out, retains its robust arterial supply and venous drainage (Fig. 1). Consequently, we designed a local flap that remains viable even when it is detached at the facial-lobule junction.

We sought to improve upon the techniques developed by Snell and Caplash [4] and Bastazini and Pianaro [5] by moving the final scar away from the free edge of the lobule and preserving as much of the lobule volume as needed to reconstruct an aesthetically pleasing earlobe.

\section{Patient selection}

We found that our technique was most suitable for large defects from earlobe gauging with diameters greater than $1 \mathrm{~cm}$ (Fig. 2A). These patients often have a residual lobular rim that does not lend itself well to simple de-epithelization or primary closure of the defect. Conversely, dilated earlobe defects that are

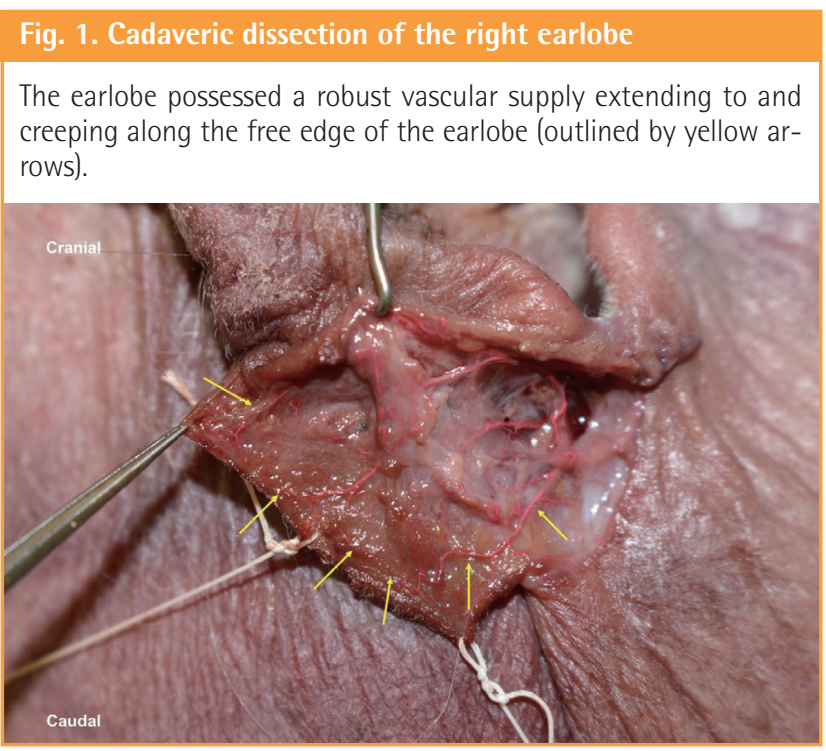

less than $1 \mathrm{~cm}$ in diameter can often be repaired with simple deepithelization of the inner surface and primary closure without significant distortion of shape or volume of the lobule (Fig. 2B).

Patients with excessively thinned-out lobules (i.e., less than 3 $\mathrm{mm}$ in width) may not carry an adequate marginal blood supply along the entire length of the lobular rim and, hence, may not be suitable for a rolling earlobe flap. Furthermore, patients who have had previous surgery, trauma, or infection of the earlobe should be assessed carefully before undergoing surgery, as the blood supply to the earlobe may be compromised.

Patients who are prone to hypertrophic scars or keloids should also be adequately warned of the eventual scars resulting from the procedure.

\section{Surgical technique}

Preoperative markings were made with the patient sitting in an upright position (Fig. 3A). Under local anesthesia (2\% lidocaine with 1:80,000 adrenaline), the inner surface of the earlobe defect was de-epithelized. The earlobe was then detached at the facial-lobule junction, erring on the side of detaching closer to the earlobe when the incision was made. This mitigated effacing the angle between the face and the lobule while concealing the eventual scar within the facial-lobule junction (Fig. 3B).

The length of the flap was trimmed according to the desired earlobe volume. This flap was then rolled onto itself similarly to a Swiss roll to create a new auricular lobule. The lateral edge of the flap was de-epithelized and the de-epithelized surfaces were brought together with polyglactin 5/0. Finally, the skin edges were meticulously opposed with nylon 6/0, first on the anterior aspect of the ear, then on the posterior aspect (Fig. 3C). When a

\section{Fig. 2. Variation in ear gauging defects}

(A) In contrast, patients with earlobe gauging defects that are greater than $1 \mathrm{~cm}$ in diameter are more suitable for reconstruction using the rolling earlobe flap. (B) Aesthetically pleasing outcomes can be simply achieved with primary closure for earlobe gauging defects that are smaller than $1 \mathrm{~cm}$ in diameter.
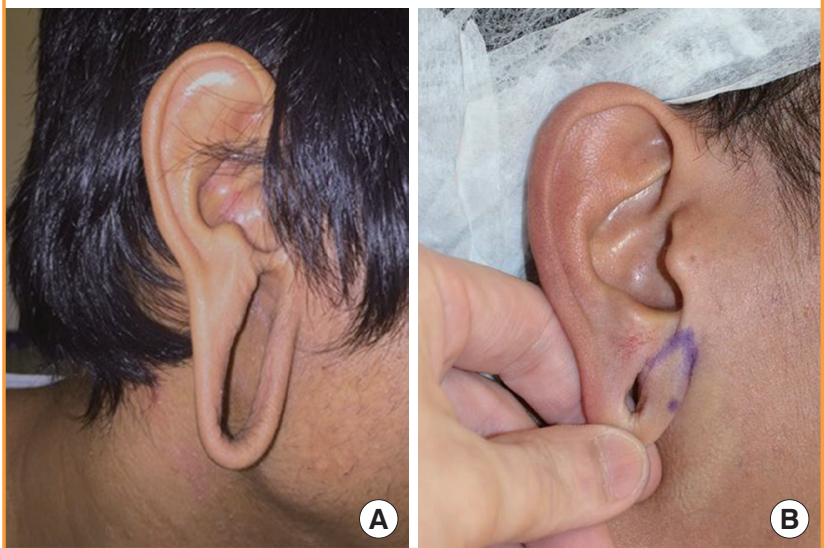


\section{Fig. 3. Surgical technique for the rolling earlobe flap}

(A) Preoperative marking. (B) Detachment at the facial-lobule junction, projected zone of de-epithelization (red dotted line), and length of redundant flap to be trimmed (shaded in blue). (C) Final positioning and suturing of rolling earlobe flap in position.
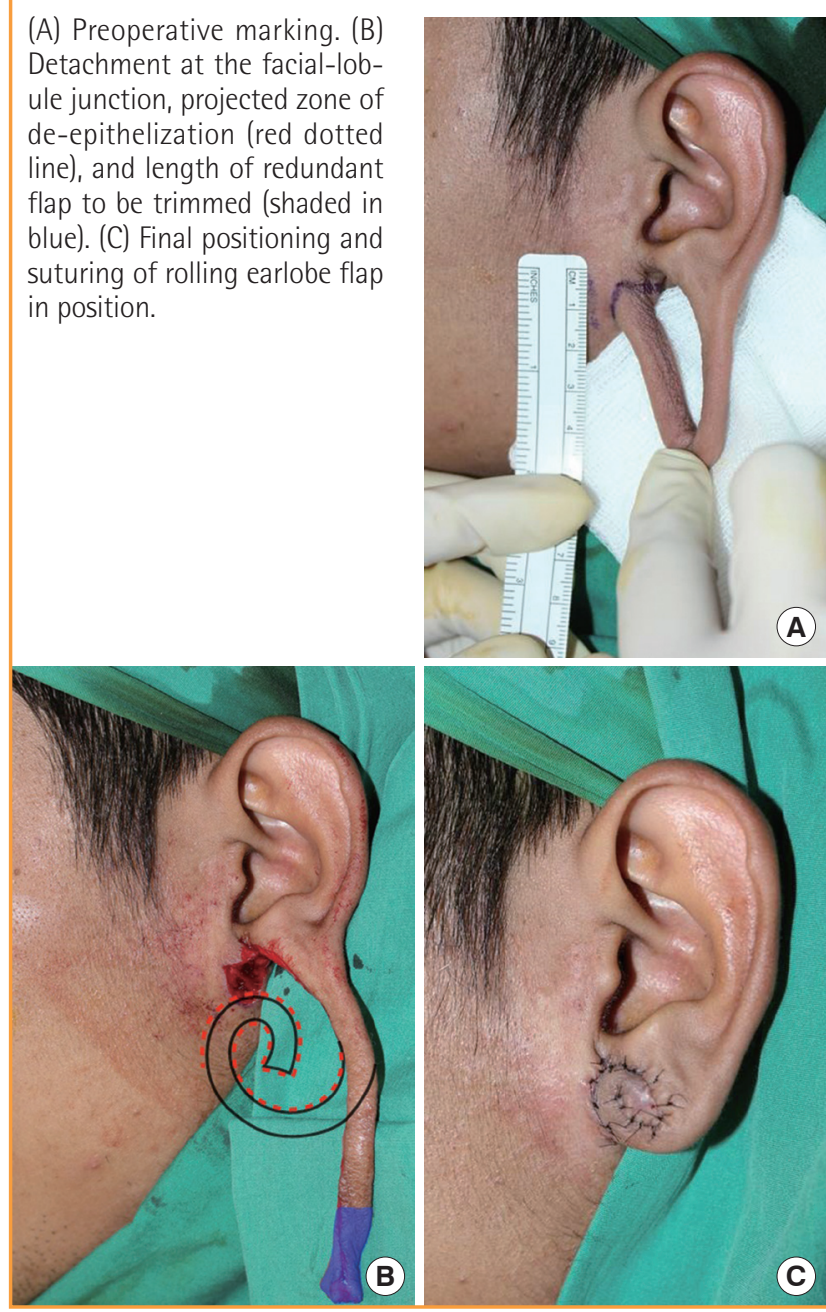

smaller lobule is desired, the flap can be simply trimmed back to a shorter length before it is rolled into position. Likewise, if an "attached" earlobe appearance is preferred, the medial aspect of the rolled-up flap is de-epithelized and sutured at the facial-lobule junction. Any asymmetry of shape or volume encountered during the first procedure should be adjusted immediately in order to minimize the need for revision through a second procedure.

\section{Results}

We repaired 6 earlobes in 3 patients using a rolling earlobe flap (Fig. 4). All patients had bilateral defects from ear gauging that ranged from 3.0 to $6.5 \mathrm{~cm}$. Surgery was performed under local anesthesia as described, and patients were discharged on the day of surgery. Skin sutures were removed on postoperative day 5 and Steri-Strip tape (3M, St. Paul, MN, USA) was used for 1 week thereafter. There were no postoperative complications of infection, wound dehiscence, flap necrosis, hypertrophic scars, or keloids.

\section{Fig. 4. Outcomes using the rolling earlobe flap}

The versatility of this flap lends itself well to earlobe gauging defects of varying sizes and allows the surgeon to control the eventual appearance of the earlobe that is reconstructed. Some Asian patients request fuller, "detached" earlobes $(A)$, while others request smaller earlobes that are "attached" at the facial-lobule junction (B). These outcomes are easily and reliably achieved regardless of the initial size of the gauging defect.
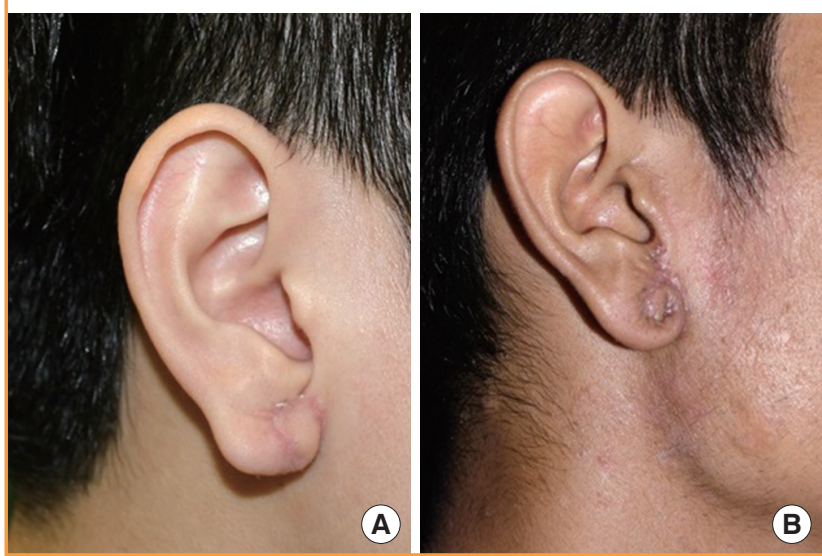

In 1 patient with a severe earlobe dilatation of $6.5 \mathrm{~cm}$, there was slight pin-cushioning of the rolling earlobe flap for the first 3 weeks following the procedure (Fig. 4B). This resolved with a scar massage and Steri-Strip taping of the reconstructed earlobes.

\section{DISCUSSION}

After extensive ear gauging, the lobule is stretched beyond its ability to recoil back into its original form. Despite the apparent expansion of tissue with ear gauging, we believe that there is actually limited gain in tissue volume of the lobule. Thus, avoidance of over-resection is important to ensure that enough tissue remains for reconstruction of the lobule. Concerns about ischemia at the tip of the flap have been raised, causing some surgeons to transect the flap around the midpoint of the lobular rim rather than at the facial-lobule junction $[4,5,8]$. This results in a scar along the free edge of the lobule. However, we have not encountered any problems with the vascularity of the rolling earlobe flap in our series.

In our review of the literature, we found that we are the first to describe the reconstruction of a dilated lobule in an Asian population. In Asia, fuller auricular lobules that are "free" at the faciallobule junction are considered a mark of prosperity. However, some patients may prefer a less prominent lobule that is attached at the facial-lobule junction. These variations in appearance are easily achieved with the technique that we described. Like other earlobe reconstruction techniques, our procedure comes with inherent risks of hypertrophic scars or keloids. 
Hence, early and aggressive scar management is required for a desirable outcome. We secured the earlobe in a desired position, minimized edema, and prevented excessive traction on the wounds with the use of postoperative Steri-Strips. The early removal of skin sutures and subsequent scar massage once the wounds had healed contributed to an optimal outcome for this procedure.

In conclusion, the rolling earlobe flap is a versatile technique that allows for an aesthetically pleasing reconstruction of the lobule with the following advantages: (1) the absence of a surgical scar on the free edge of the lobule, (2) preservation of lobule volume, and (3) a highly customizable technique that allows for the creation of lobules of various shapes and volumes.

\section{PATIENT CONSENT}

The patient provided written informed consent for the publication and the use of their images.

\section{REFERENCES}

1. Zeiderman MR, Kelishadi SS, Tutela JP, et al. Double opposing perpendicular linear repair of gauge ear-piercing deformity: a new technique and literature review. Eplasty 2014;14:e31.
2. Henderson J, Malata CM. Surgical correction of the expanded earlobe after ear gauging. Aesthetic Plast Surg 2010;34: 632-3.

3. Collins J, Harvey J, Hilinski JM. Reconstructing the gauge earlobe defect. JAMA Facial Plast Surg 2015;17:144-8.

4. Snell BJ, Caplash Y. A novel way to repair the earlobe after ear-gauging. J Plast Reconstr Aesthet Surg 2013;66:140-1.

5. Bastazini I Jr, Pianaro C. New approach to repair of expanded earlobe. Dermatol Surg 2013;39:1127-9.

6. de la Sotta P, Paredes N, Lasalle MA. Repair of dilated earlobe due to plug piercing. Dermatol Surg 2010;36:1621-3.

7. Williams AM, Majumder S. Earlobe reconstruction following plug and tunnel piercing. J Plast Reconstr Aesthet Surg 2010;63:e294-5.

8. Ehrl D, Erne HC, Erwin F. Reconstruction of the earlobe while preserving its volume following tunnel and plug piercing. J Plast Reconstr Aesthet Surg 2015;68:590-2.

9. Zilinsky I, Cotofana S, Hammer N, et al. The arterial blood supply of the helical rim and the earlobe-based advancement flap (ELBAF): a new strategy for reconstructions of helical rim defects. J Plast Reconstr Aesthet Surg 2015;68: 56-62.

10. Park C, Lineaweaver WC, Rumly TO, et al. Arterial supply of the anterior ear. Plast Reconstr Surg 1992;90:38-44. 